\title{
Effects of Aging on Nasal Mucociliary Clearance
}

\author{
${ }^{1}$ J yoti Yadav, ${ }^{2}$ Rupender K Ranga, ${ }^{3}$ agat Singh \\ ${ }^{1}$ P rofessor, Department of Physiology, Pt. BD S harma Postgraduate Institute of Medical Sciences, Rohtak, Haryana, India \\ ${ }^{2}$ Consultant, Bharat ENT and Endoscopy Hospital, Rohtak Gate, Bhiwani, Haryana, India \\ ${ }^{3}$ Assistant Professor, Department of Otorhinolaryngology, Pt. BD Sharma Postgraduate Institute of Medical Sciences, Rohtak, Haryana, India
}

Correspondence: Rupender K Ranga, Bharat ENT and Endoscopy Hospital, Rohtak Gate, Bhiwani-127021, Haryana, India e-mail: bharatentbwn@ sify.com, rupenderentbwn@yahoo.com

\begin{abstract}
Nasal mucociliary clearance is a primary physiological defense mechanism of upper as well as lower respiratory tract, and thus maintains the state of health of the respiratory tract. Any disturbance in number or movement of cilia and change in character or amount of mucus production leads to an altered nasal mucociliary clearance. The present study was undertaken to study the effect of aging on nasal mucociliary clearance.

Nasal mucociliary clearance time was assessed in 240 normal subjects, 120 males and 120 females, which were divided in six groups, each comprising of one decade starting from 11 to 20 years. The mean nasal mucociliary clearance time ranged 7.34 to 14.48 minutes in males and 7.36 to 15.38 minutes in females in different groups (A-F). There was no statistical difference between males and females in each group ( $p>0.05)$. The nasal mucociliary clearance time was not found to be increased till 5 th decade $(p=0.05)$. However, the nasal mucociliary clearance time was found to be increased in 6 th and 7 th decades (group E\&F) significantly $(p \leq 0.001$ ), which was more marked in group $F$. This may explain the increased susceptibility of elderly to sinusitis chronic bronchitis, deep lung infections and increased incidence of pneumonia.
\end{abstract}

Keywords: Mucociliary clearance, Kartagener's syndrome, Andersen's saccharin method.

\section{INTRODUCTION}

$N$ asal mucociliary clearance is a primary physiological defense mechanism of upper as well as lower respiratory tract, and thus maintains the state of heal th of the respiratory tract. The vital part of this mechanism is an adequate quantity of mucus with appropriate rhinological qualities and adequately functioning cilia, which beat in metachronous fashion towards nasopharynx. ${ }^{1}$ A ny disturbance in number or movement of cilia and change in character or amount of mucus production leads to an altered nasal mucociliary clearance.

The respiratory epithelium is essential for defense of the airways against inhaled pathogens. ${ }^{2}$ When inhaled pollutants and pathogens reach the upper respiratory tract and adhere to surface mucus, they are conveyed by mucociliary clearance to the nasopharynx and periodically swallowed. As the age advances, the ciliary function gets slowed down and becomes more prone to secondary respiratory infection as is also seen in certain diseases like K artagener's syndrome, cystic fibrosis, asthma, bronchiectasis, chronic bronchitis and sinusitis. ${ }^{3-6}$ A lthough, mucociliary clearance might decline with aging, little is known about the effect of aging on nasal mucociliary clearance. The present study was undertaken to study the effect of aging on nasal mucociliary clearance.

\section{MATERIALS AND METHODS}

The present study was conducted on 240 normal persons of either sex in equal numbers in age group of 11 to 70 years. The study sample was chosen using random sampling technique with adequate numbers of participants without any ENT or lower respiratory tract pathology. They were divided into six groups $A, B, C, D, E$ and $F$ comprising of $11-20,21-30,31-40,41-50,51-60$ and $61-70$ years respectively. Detailed history was obtained and routineENT examination was carried out. The diseases which are known to affect nasal mucociliary clearance like nasal polyps, deviated nasal septum, foreign bodies in nose, enlarged adenoids, allergic and atrophic rhinitis along with lower airway obstructive condition like bronchial asthma and chronic pulmonary diseases, where mucociliary clearance is known to be impaired, were also excluded from the study. The person on drugs like bromhexine that are also known to affect nasal mucociliary clearance and those who smoked were also excluded from the study

Nasal mucociliary clearance was evaluated by means of A ndersen's saccharin method, ${ }^{7}$ which is simple, quick, non-invasive and serves as a valuable screening test.

A saccharin particle (1.5 mm diameter) was carefully placed on the floor of nasal cavity about $1 \mathrm{~cm}$ behind the anterior end of the inferior turbinate. The patients were asked 
not to sniff, sneeze, smoke, eat or drink during the test and avoid deep breathing. They were asked to swallow every 30 seconds and to report the first change in their sensation of taste. The time taken by the patients from placement of particle to the perception of the sweet taste in the pharynx was noted. The nature of the particle was not disclosed to the patient to ensure the reliability of the procedure. The test was repeated in the other nostril and the mean of the two sides was taken as mucociliary time. This was done to exclude the effect, if any, of nasal cycle on the mucociliary time. The results were statistically analyzed by using ' $z$ ' test.

\section{RESULTS}

The subjects were divided into six groups and each group consists of 40 healthy age and sex matched person. Group A (11-20 years), B (21-30 years), C (31-40 years), D (4150 years), $E$ (51-60 years) and $F$ (61-70 years) having mean age 16.4:16.6, 24.4:24.2, 34.0:34.6, 44.4:44.2, 54.8:54.6 and 66.0:65.0 in males and females respectively. $M$ ale and female ratio was almost equal in various groups (Table 1 ).

\section{Table 1: Age and sex distribution of subjects}

\begin{tabular}{lccccc} 
Groups & \multicolumn{2}{c}{ Mean age ( in years) } & & \multicolumn{2}{c}{ No. of sex } \\
\cline { 2 - 3 } \cline { 5 - 6 } & Male & F emale & & M ale & F emale \\
\hline A (11-20 years) & 16.4 & 16.6 & & 20 & 20 \\
B (21-30 years) & 24.4 & 24.2 & & 20 & 20 \\
C (31-40 years) & 34.0 & 34.6 & & 20 & 20 \\
D (41-50 years) & 44.4 & 44.2 & & 18 & 22 \\
E (51-60 years) & 54.8 & 54.6 & & 28 & 12 \\
F (61-70 years) & 66.0 & 65.0 & & 18 & 22 \\
\hline
\end{tabular}

The mean value of nasal mucociliary clearance time in group A was 7.34 minutes in males and 7.36 minutes in females, group B was 8.04 minutes in males and 7.48 minutes in females, group $C$ was 7.14 minutes in males and 7.56 minutes in females, group D was 8.42 minutes in males and 9.54 minutes in females, group $E$ was 11.58 minutes in males and 11.48 minutes in females, group $F$ was 11.48 minutes in males and 15.38 minutes in females. The nasal mucociliary clearance time showed insignificant difference between two sexes in all the groups. The mean value of nasal mucociliary clearance when compared $A$ vs $B, B$ vs $C$, $C$ vs $D, D$ vs $E$ was found to be insignificant $(p=0.05)$, however, $E$ vs $F$ was found statistically significant $(p=$ 0.001 ). When group $A$ vs $B, A$ vs $C, A$ vs $D$ were compared, it was statistically insignificant $(p=0.05)$, but when $A$ vs $E$ and $A$ vs $F$ were compared it was statistically highly significant $(p=0.001)$ (Table 2 ).
Table 2: Nasal mucociliary clearance in various groups

\begin{tabular}{lcc} 
Groups & \multicolumn{2}{c}{ Nasal mucociliary clearance in minutes } \\
\cline { 2 - 3 } & Male & F emale \\
\hline A (11-20 years) & 7.34 & 7.36 \\
B (21-30 years) & 8.04 & 7.48 \\
C (31-40 years) & 7.14 & 7.56 \\
D (41-50 years) & 8.42 & 9.54 \\
E (51-60 years) & 11.58 & 11.48 \\
F (61-70 years) & 14.48 & 15.38 \\
\hline
\end{tabular}

\begin{tabular}{lll}
\hline Groups & Male & Female \\
\hline$A$ vs $B$ & $p \geq 0.05$ & $p \geq 0.05$ \\
$B$ vs $C$ & $p \geq 0.05$ & $p \geq 0.05$ \\
$C$ vs $D$ & $p \geq 0.05$ & $p \geq 0.05$ \\
$D$ vs $E$ & $p \geq 0.05$ & $p \geq 0.05$ \\
$E$ vs $F$ & $p \leq 0.001$ & $p \leq 0.001$ \\
$A$ vs $C$ & $p \geq 0.05$ & $p \geq 0.05$ \\
$A$ vs $D$ & $p \geq 0.05$ & $p \geq 0.05$ \\
$A$ vs $E$ & $p \leq 0.001$ & $p \leq 0.001$ \\
$A$ vs $F$ & $p \leq 0.001$ & $p \leq 0.001$ \\
\hline
\end{tabular}

\section{DISCUSSION}

$\mathrm{N}$ asal mucociliary clearance cleans airways of its own secreted mucus, together with inhaled pollutants as well as pathogens trapped in it. In turns, cough serves as a backup system when nasal mucociliary clearance function is impaired. A ging is a complex process associated with the 'maturation and decline of a given function,' however in aged, impaired nasal mucociliary clearance functions are pathogenic factor for development of chronic bronchitis. ${ }^{8}$ Salivation and swallowing are protective mechanism in oral cavity, same way as the mucociliary clearance is in the respiratory tract.

In present study, the mean value of nasal mucociliary clearance time was 7.35 minutes in 11 to 20 years age group. The mean value of nasal mucociliary clearance time ranging from 5.7 to 8.55 minutes has been reported in healthy adults. ${ }^{9}$ In group B, nasal mucociliary clearance time was 7.76 minutes, group $C$ was 7.35 minutes and group $D$ was 8.98 minutes. The mean value of nasal mucociliary clearance time at Rohtak, Chandigarh, Calcutta and Nagpur are reported to be $5.7,5.06,4.4$ and 7.1 minutes respectively in healthy subjects, which is comparable to our study as the difference of tw o minutes between two studies is considered as insignificant. ${ }^{10-13}$

The mean value of nasal mucociliary clearance in 51 to 60 years age (group E) is 11.53 minutes and 14.93 minutes in group $F$, whereas in western countries a wide range of 
nasal mucociliary clearance time is reported from 3.3-35 minutes in adults. ${ }^{14,15}$ In present studies, the mean value of nasal mucociliary clearance time has been reported to be 8.2 minutes in adults, however, there can be considerable variation in value at different places even in India, depending on habit, habitat, climate and difference in facial configuration. ${ }^{16}$

The mean value of nasal mucociliary clearance time in group $E$ and $F$ was compared with group $A$ to $D$. It was found to be significantly higher. It is based on possible hypothesis that decreased pulmonary function and nasal mucociliary function might be a part of normal aging in heal thy subjects, as was observed in the present study and is associated with high prevalence of respiratory symptoms among otherwise healthy elderly. ${ }^{8,17}$

Increased susceptibility of elderly to deep lung infections and sinusitis may be associated with impaired mucociliary clearance. F urther bacterial flora in oral cavity and impaired salivary clearance also contribute to pathogenic colonization. ${ }^{18}$ Some studies also defined that aging influence breathing pattern and airway geometry, however once the lungs are mature, the available evidence indicate that deposition to the aging lung does not change over the time as a factor of age alone. ${ }^{19}$

Physiological factors, such as age, sex, posture, sleep and exercise are reported to influence nasal mucociliary clearance due to change in the cilia, mucus or periciliary layer or combination of these. ${ }^{8}$ Environmental pollution is suspected to have a depressant effect on nasal mucociliary clearance depending on different factors, such as pollutant concentration and duration of exposure. M ost studies focus on sulphur dioxide, sulphuric acid, nitrogen dioxide and ozone. ${ }^{17} \mathrm{~A}$ s the age advances, there is cumulating effect of these pollutants on nasal mucociliary clearance, however, this manifest only in the 6th decade onwards as was observed by us. This may explain the facts that elderly are more prone to pneumonia as compared to young adults.

\section{REFERENCES}

1. Waguespack R. M ucociliary clearance pattern following endoscopic sinus surgery. L aryngoscope 1995;105:1-40.

2. Yager J, Chen T, Dulfano M J. M easurement of frequency of ciliary beats of human respiratory epithelium. Chest 1978;73: 627-33.
3. Goodman RM , Y ergin BM , L anda JF, Golinvaux M H, Sackner $M A$. Relationship of smoking history and pulmonary function tests to tracheal mucus velocity in non smokers, young smoker, ex-smoker and patients suffering from bronchitis. A m Rev R espir Dis 1978;117:205-14.

4. Ruthland J, Cole PJ. Nasal mucociliary clearance and ciliary beat frequency in cystic fibrosis compared with sinusitis and bronchiectasis. Thorax 1981;36:654-81.

5. Stanley PJ, W ilson R, Greenstone MA, M akay IS, Cole PJ. A bnormal mucociliary clearance in patients with rhinitis and its relationship to concomitant chest diseases. $\mathrm{Br} J$ Dis Chest 1985;79:77-82.

6. Wig U, Jindal NK, Goel H, Chawla RK, Y adav SPS. Nasal mucous clearance in nasal and paranasal sinus disorders. Indian J Chest Dis A II Sci 1988;30:176-80.

7. A ndersen $I$, L undquist $G$, J ensen $P L$, Philipson $K$, Proctor DF. Nasal clearance in monozygotic twins. A m Rev Respir Dis 1978; 110:301-05.

8. Gerald CS. Deposition and clearance: Unique problems in the proximal airway and oral cavity in the young and elderly. Resp Physio 2001;128:33-38.

9. Ranga RK, Singh J, Gera A, Y adav J. Nasal mucociliary clearance in adenotonsillar hypertrophy. Indian J Paediatr 2000; 67:651-52.

10. Y adav J, Ranga RK, Singh J, Gathwala G. Nasal mucociliary clearance in healthy children in a tropical country. Int J Paedr Otorhinolaryngol 2001;57:21-24.

11. Mehra $Y N$, Mann SBS, M ehra S, Verma A, Mittal A. Cryosurgery in vasomotor rhinitis: A $n$ analysis of 156 patients. Indian J Otolaryngol 1996;42:95-98.

12. Mukherjee Al. Human nasal mucus flow rates. Indian J Otolaryngol 1977;29:174-75.

13. Golhar S, A rora M M L. The effect of cryodestruction of vidian nasal branches on nasal mucus flow in vasomotor rhinitis. Indian J Otolaryngol 1981;97:12-14.

14. Stanley P, M acwilliam L, G reenstone M, M ackay I, Cole P. Efficiency of saccharin test for screening to detect abnormal mucociliary clearance. $\mathrm{Br}$ J Dis Chest 1984;78:62-65.

15. Puchelle E, A ug F, Pham QT, B ertrend A. Comparison of three method for measuring nasal mucociliary clearance in man. A cta Otolaryngol 1981;91:297-303.

16. $Y$ adav J, V erma A, Singh J. Study on nasal mucus clearance in patients of perennial allergic rhinitis. Indian J Allergy A sthma Immunol 2003;17:89-91.

17. Houtmeyers E, Gosselink R, Gayan- Ramirez G, Decramer M . Regulation of mucociliary clearance in health and diseases. Eu Respir J 1999;13:1177-88.

18. Gleeson DCL. Oropharyngeal swallowing and aging: A review. J Commun Disord 1999;32:373-96.

19. Holfmann W, M artonen TB, Graham RC. Predicted deposition of non hygroscopic aerosols in human lungs as a function of age. J A erosol M ed 1989;1:49-68. 